ORIGINAL ARTICLE

\title{
Effect of contraction frequency on energy expenditure and substrate utilisation during upper and lower body exercise
}

\author{
J Kang, J R Hoffman, M Wendell, H Walker, M Hebert
}

Br J Sports Med 2004;38:31-35. doi: 10.1136/bjsm.2002.002121

See end of article for authors' affiliations

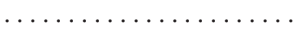

Correspondence to:

Dr Kang, Department of

Health and Exercise

Science, The College of

New Jersey, 234 Packer

Hall, 2000 Pennington

Road, Ewing, NJ 08628,

USA; kang@tcnj.edu

Accepted

5 November 2002
Objective: To examine the effect of contraction frequency on energy expenditure and substrate utilisation during upper (UE) and lower (LE) body exercise.

Methods: Twenty four college students were recruited: 12 were tested on an arm ergometer, and the other 12 were tested on a leg ergometer. Each subject underwent three experimental trials on three separate days, and the three trials were presented in a randomised order. Each trial consisted of 10 minutes of arm cranking or leg cycling at 40,60 , or $80 \mathrm{rev} / \mathrm{min}$, with power output being kept constant at $50 \mathrm{~W}$. Steady state oxygen uptake $\left(\mathrm{VO}_{2}\right)$ and respiratory exchange ratio (RER) were measured during each exercise. Energy expenditure was calculated from the steady state $\mathrm{VO}_{2}$ adjusted for substrate metabolism using RER. Carbohydrate and fat oxidation were calculated from $\mathrm{VO}_{2}$ and $\mathrm{RER}$ based on the assumption that protein breakdown contributes little to energy metabolism during exercise.

Results: Energy expenditure was greater $(p<0.05)$ at $80 \mathrm{rev} / \mathrm{min}$ than at $40 \mathrm{rev} / \mathrm{min}$. No difference was found between 40 and $60 \mathrm{rev} / \mathrm{min}$ and between 60 and $80 \mathrm{rev} / \mathrm{min}$ during both UE and LE. During LE, carbohydrate oxidation was also higher at $80 \mathrm{rev} / \mathrm{min}$ than at $40 \mathrm{rev} / \mathrm{min}$, whereas no difference in fat oxidation was found among all three pedal rates. During UE, no speed related differences in either carbohydrate or fat utilisation were observed.

Conclusions: Pedalling at a greater frequency helped to maximise energy expenditure during exercise using UE or LE despite an unchanging power output. Whereas contraction frequency affects energy expenditure similarly during both UE and LE, its impact on carbohydrate utilisation appears to be influenced by exercise modality or relative exercise intensity.
$P$ ower output of any given activity is determined by both the speed at which movement takes place and the force that is generated by the exercising muscle. One can maintain a constant power output even when speed is varied. However, despite an unchanging power output, a change in speed and thus force has been shown to affect many physiological responses including metabolic efficiency, ${ }^{1-7}$ oxygen deficit, ${ }^{8}$ lactate threshold, ${ }^{9}$ and aerobic capacity. ${ }^{8}$

Much of the pertinent literature has been related to movement economy or athletic performance. Whether a change in speed would affect patterns of energy expenditure and substrate utilisation has not been thoroughly investigated. This is an intriguing question given that some commonly used exercise ergometers are equipped with a servo mechanism with which speed of movement can be selected without a concurrent change in workload. Cycling at a higher pedal rate has been considered to not only recruit more motor units, but also elicit a higher concentration of blood lactate despite an unchanging workload. ${ }^{9}$ In this context, it may be speculated that more energy would be expended and carbohydrate used if exercise were performed at a fast velocity concomitant with less muscular tension. This hypothesis, however, remains to be tested, as Hagan et al ${ }^{10}$ has shown a greater respiratory exchange ratio (RER) during cycling of a constant load at 60 as compared with $90 \mathrm{rev} / \mathrm{min}$. In addition, Ahlquist et $a l^{11}$ showed greater glycogen utilisation of type II muscle fibres after cycle exercise of $85 \% \mathrm{VO}_{2} \mathrm{MAX}$ at 50 than at $100 \mathrm{rev} / \mathrm{min}$.

Therefore the primary purpose of this study was to examine whether and how contraction frequency affects energy expenditure and substrate utilisation during exercise. As a similar power output represents a much greater relative strain during exercise using upper body musculature consisting of more fast twitch muscle fibres, ${ }^{12}{ }^{13}$ the second- ary purpose of this study was to examine whether the relation between contraction frequency and energy metabolism is affected by exercise modality or relative exercise intensity.

\section{METHODS}

\section{Subjects}

Twenty four college aged volunteers ( 12 men, 12 women) served as subjects. None had been trained using either the arm or cycle ergometer. Twelve (seven men, five women) were tested on a cycle ergometer, and the remaining 12 (five men, seven women) were tested on an arm ergometer. All subjects were healthy and free from any orthopaedic injury at the time of the investigation. They were informed of the purpose of the experiment and gave their written consent to participate. All experimental procedures were evaluated and approved by the Institutional Review Board for Human Subjects Experimentation at The College of New Jersey. Table 1 presents the physical and physiological characteristics of the subjects.

\section{Experimental design}

Subjects within each group completed a peak oxygen uptake $\left(\mathrm{VO}_{2}\right.$ peak) test and three submaximal exercise trials on four separate laboratory visits. All subjects were instructed to have eaten a minimum of three hours before each laboratory visit. The $\mathrm{VO}_{2}$ peak test was always conducted before the three submaximal exercise trials. A minimum of 48 hours sepa-

Abbreviations: $H R$, heart rate; $L E$, lower body exercise; RER, respiratory exchange ratio; $\mathrm{UE}$, upper body exercise; $\mathrm{VCO}_{2}$, carbon dioxide production; $\mathrm{VE}$, expiratory ventilation; $\mathrm{VO}_{2}$, oxygen uptake 


\begin{tabular}{|c|c|c|c|}
\hline & Men & Women & Total \\
\hline \multicolumn{4}{|l|}{ Upper body exercise } \\
\hline Number & 5 & 7 & 12 \\
\hline Age (years) & $21(3)$ & $20(1)$ & $21(2)$ \\
\hline Height $(\mathrm{m})$ & $1.77(0.08)$ & $1.69(0.1)$ & $1.71(0.12)$ \\
\hline Body mass (kg) & $83(4)$ & $66(5)$ & 77 (9) \\
\hline $\operatorname{BMI}\left(\mathrm{kg} / \mathrm{m}^{2}\right)$ & $27.0(2.5)$ & $22.5(1.3)$ & $25.5(2.7)$ \\
\hline $\mathrm{VO}_{2}$ peak (litres/min) & $2.29(0.50)$ & $2.11(0.31)$ & $2.21(0.41)$ \\
\hline HRpeak (beats/min) & $170(15)$ & $175(12)$ & $172(13)$ \\
\hline \multicolumn{4}{|l|}{ Lower body exercise } \\
\hline Number & 7 & 5 & 12 \\
\hline Age (years) & $21(1)$ & $23(5)$ & $22(4)$ \\
\hline Height $(\mathrm{m})$ & $1.80(0.03)$ & $1.62(0.06)$ & $1.70(0.11)$ \\
\hline Body mass (kg) & $82(9)$ & $59(8)$ & 66 (12) \\
\hline $\mathrm{BMI}(\mathrm{kg} / \mathrm{m})$ & $24.9(2.3)$ & $23.1(2.3)$ & $23.7(2.3)$ \\
\hline $\mathrm{VO}_{2}$ peak (litres/min) & $3.10(0.45)$ & $2.35(0.35)$ & $2.79(0.44)$ \\
\hline HRpeak (beats/min) & $186(13)$ & $184(12)$ & 185 (13) \\
\hline
\end{tabular}

rated each visit, and all four visits were completed within a three week period. The three submaximal exercise trials were conducted at the same time of day and were presented in a randomised and counterbalanced order. During each submaximal exercise trial, subjects underwent a 10 minute arm or leg ergometry test during which both metabolic and cardiorespiratory variables were measured once the steady state had been achieved.

\section{Exercise trials}

Equipment set up

The upper body exercise (UE) was conducted on a modified Monark cycle ergometer (Monark 864, Monark Exercise AB, Varberg, Sweden). The sprocket of the ergometer was adjusted so that the crank axle was at shoulder level. Selection of this leg ergometer for arm use was to equate the size of flywheel so that the flywheel per se would not confound the results. ${ }^{14}$ The lower body exercise (LE) was conducted on a standard Monark cycle ergometer (Monark 828E). For both UE and LE, subjects were positioned so that their respective limbs were extended but not locked when the pedal was at its farthest end from the subjects.

\section{$\mathrm{VO}_{2}$ peak tests}

During the arm and leg $\mathrm{VO}_{2}$ peak tests, subjects performed a continuous incremental protocol that was similar to those reported previously. ${ }^{15}$ The protocol for UE was designed by Sawka et al, ${ }^{17}$ and that for LE was developed by Åstrand. ${ }^{18}$ The test was preceded by a 10 minute warm up period. The initial power output was $25 \mathrm{~W}$ for the arm test and $50 \mathrm{~W}$ for the leg test. For both tests, power output was increased by $25 \mathrm{~W}$ every two minutes. The pedal rate was set at $50 \mathrm{rev} / \mathrm{min}$ for both tests, with the cadence guided by a metronome. All subjects were verbally encouraged to continue exercise until volitional exhaustion. A plateau in $\mathrm{VO}_{2}$ was not generally observed in the arm tests. This was also observed in our previous study, ${ }^{15}$ the reason being that the muscle mass involved is small and muscular fatigue normally occurs before the cardiorespiratory system reaches its peak. In this case, the termination criterion is simply when the subject fails to maintain a target crank frequency for 15 seconds. However, the plateau as well as RER greater than 1.1 were shown in all of the leg tests. During both arm and leg tests, $\mathrm{VO}_{2}$ and heart rate (HR) were recorded every 20 seconds, and $\mathrm{VO}_{2}$ peak was determined by averaging the two consecutive highest measurements.

\section{Submaximal exercise trials}

During each submaximal exercise trial, subjects performed a 10 minute steady state exercise at 40,60 , or $80 \mathrm{rev} / \mathrm{min}$. The target pedal rate for each trial was maintained by using a metronome. The power output was set at $50 \mathrm{~W}$ for both types of exercise; this intensity was chosen to ensure that all subjects were able to achieve a steady state especially during the arm exercise. Beginning at the 8th minute of each exercise, $\mathrm{VO}_{2}$, carbon dioxide production $\left(\mathrm{VCO}_{2}\right)$, expiratory ventilation (VE), RER, and HR were recorded at 20 second intervals, and the data then averaged over the next two minute period. Measurements of these variables were also taken at rest before the exercise during the first visit to the laboratory.

\section{Measurements}

$\mathrm{VO}_{2}, \mathrm{VCO}_{2}, \mathrm{VE}$, and RER were determined using a two way $\mathrm{T}$ shape breathing valve (2700 series; Hans Rudolph, Inc, Kansas City, Missouri, USA) and an open circuit respiratory metabolic system (Metabolic Measurement Cart 2900; SensorMedics, Inc, Yorba Linda, California, USA). HR was measured using a wireless HR monitor (Pacer; Polar CIC, Inc, Port Washington, New York, USA), which updated values every five seconds. All trials were conducted in the laboratory where the mean barometric pressure and laboratory temperature were 755 (2) $\mathrm{mm} \mathrm{Hg}$ and 23.2 (1.2) ${ }^{\circ} \mathrm{C}$ respectively.

\section{Calculations}

Energy expenditure during exercise was calculated from the steady state $\mathrm{VO}_{2}$ adjusted for substrate metabolism using the RER. ${ }^{16}$ The rates of carbohydrate and fat oxidation were calculated from $\mathrm{VO}_{2}$ and RER based on the assumption that protein breakdown contributes little to energy metabolism during exercise.

\section{Statistical analysis}

Statistical analyses were performed separately for each mode of exercise. Within each mode of ergometry, a one way analysis of variance with repeated measures was used to compare all dependent variables including those measured and calculated across three levels of pedal frequency. A significant $F$ ratio was followed by a post hoc analysis using the Sheffé procedure. All statistical analyses were performed using SPSS software (SPSS Inc, Chicago, Illinois, USA). 
Table 2 Cardiorespiratory and metabolic responses at rest and during upper and lower body exercise of various contraction frequencies

\begin{tabular}{|c|c|c|c|}
\hline & $40 \mathrm{rev} / \mathrm{min}$ & $60 \mathrm{rev} / \mathrm{min}$ & $80 \mathrm{rev} / \mathrm{min}$ \\
\hline \multicolumn{4}{|l|}{ Upper body exercise } \\
\hline $\mathrm{VO}_{2}$ (litres $/ \mathrm{min}$ ) & $1.32(0.04)$ & $1.35(0.04)$ & $1.54(0.05)^{*}$ \\
\hline HR (beat $/ \mathrm{min}$ ) & $122(6)$ & $125(5)$ & $134(6)^{*}$ \\
\hline VE (litres/min) & $38.0(2.1)$ & $28.9(1.5)$ & $46.5(3.4)^{*}$ \\
\hline RER & $0.96(0.02)$ & $0.98(0.02)$ & $0.94(0.02)$ \\
\hline$\% \mathrm{VO}_{2}$ peak & 60 (3) & $61(4)$ & $70(4)^{*}$ \\
\hline \%HRpeak & 70 (4) & $72(4)$ & $78(4)^{*}$ \\
\hline \multicolumn{4}{|l|}{ Lower body exercise } \\
\hline $\mathrm{VO}_{2}$ (litres/min) & $0.99(0.01)$ & $1.00(0.02)$ & $1.16(0.02)^{*}$ \\
\hline $\mathrm{HR}$ (beat $/ \mathrm{min}$ ) & $108(3)$ & $109(3)$ & $119(4)^{*}$ \\
\hline$V_{E}$ (litres/min) & $24.3(1.0)$ & $24.8(0.8)$ & $29.8(1.1)^{*}$ \\
\hline RER & $0.79(0.01)$ & $0.83(0.01)$ & $0.84(0.01)$ \\
\hline$\% \mathrm{VO}_{2}$ peak & $35(2)$ & $36(3)$ & $42(3)^{*}$ \\
\hline \%HRpeak & 58 (3) & 59 (3) & $65(4)^{*}$ \\
\hline $\begin{array}{l}\text { Values are mean (S } \\
{ }^{*} \text { Significantly differe } \\
\mathrm{VO}_{2}, \text { Oxygen uptak }\end{array}$ & . & 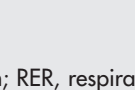 & 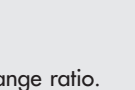 \\
\hline
\end{tabular}

\section{RESULTS}

\section{Upper body exercise}

During UE, no differences in $\mathrm{VO}_{2}, \mathrm{HR}, \mathrm{VE}, \% \mathrm{VO}_{2}$ peak, and \%HRpeak were observed between 40 and $60 \mathrm{rev} / \mathrm{min}$ (table 2). However, all these variables were higher $(p<0.05)$ at $80 \mathrm{rev} /$ min than at either 40 or $60 \mathrm{rev} / \mathrm{min}$. This greater physiological response at the higher pedal rate was not observed for RER. In fact, there was a trend toward a higher RER at 40 and $60 \mathrm{rev} / \mathrm{min}$ than at $80 \mathrm{rev} / \mathrm{min}$.

The total energy expenditure was higher $(\mathrm{p}<0.05)$ at $80 \mathrm{rev} / \mathrm{min}$ than at $40 \mathrm{rev} / \mathrm{min}$, whereas no difference was found between 40 and $60 \mathrm{rev} / \mathrm{min}$ and between 60 and $80 \mathrm{rev} / \mathrm{min}$ (fig 1). However, both carbohydrate and fat oxidation remained the same across all three pedal rates.

\section{Lower body exercise}

During $\mathrm{LE}, \mathrm{VO}_{2}, \mathrm{HR}, \mathrm{VE}, \% \mathrm{VO}_{2}$ peak, and \%HRpeak were higher $(p<0.05)$ at $80 \mathrm{rev} / \mathrm{min}$ than at either 40 or $60 \mathrm{rev} /$ min. These changes were similar to those observed during UE, except that RER at $40 \mathrm{rev} / \mathrm{min}$ remained marginally the lowest among the three pedal rates (table 2 ).

The total energy expenditure was also higher $(\mathrm{p}<0.05)$ at $80 \mathrm{rev} / \mathrm{min}$ than at $40 \mathrm{rev} / \mathrm{min}$, whereas no difference was found between 40 and $60 \mathrm{rev} / \mathrm{min}$ and between 60 and $80 \mathrm{rev} / \mathrm{min}$ (fig 2). Unlike that observed during UE, carbohydrate oxidation was also higher $(\mathrm{p}<0.05)$ at $80 \mathrm{rev} / \mathrm{min}$ than at $40 \mathrm{rev} / \mathrm{min}$, while no differences in fat oxidation were observed among the three pedal rates.
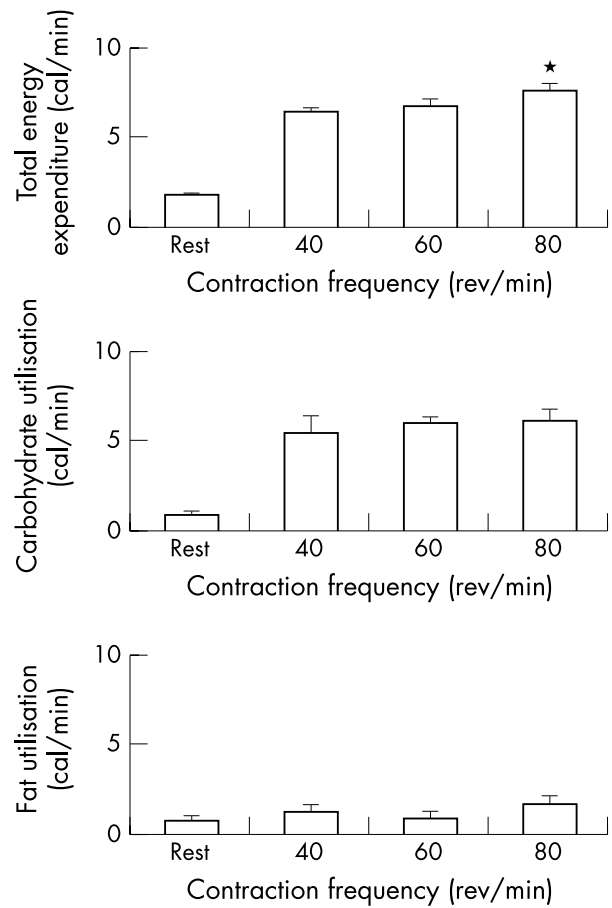

Figure 1 Total energy expenditure and carbohydrate and fat utilisation at rest and during upper body exercise at various pedal frequencies. Resting values are provided as a reference and were not used in the statistical analysis. ${ }^{*}$ Significantly higher at 80 than at $40 \mathrm{rev} / \mathrm{min}$, $\mathrm{p}<0.05$. 


\section{DISCUSSION}

Pedal frequency as a mechanical constituent of power output reflects the velocity at which movement is taking place. Previous studies have shown the effect of this speed component on physiological processes while power output is held constant. However, many of these studies have either focused on movement economy or athletic performance. With the advance of technology, there has been increased use of exercise apparatus equipped with a servo mechanism which enables the subject to choose a speed without changing workload in both recreational and clinical settings. Although this feature provides exercisers with additional options of conducting a workout, it raises questions about how speed of movement can affect energy metabolism and what would be the optimum speed for energy expenditure. We therefore examined whether and how contraction frequency affects energy expenditure as well as carbohydrate and fat oxidation during both UE and LE. This was accomplished by having subjects pedal on either an arm or a leg ergometer at a constant power output but various frequencies while their metabolic responses were measured using indirect calorimetry.

We found a greater energy expenditure at $80 \mathrm{rev} / \mathrm{min}$ than at $40 \mathrm{rev} / \mathrm{min}$ during both UE and LE despite an unchanging power output and a concomitant reduction in brake resistance. This speed related increase in total energy expended during both UE and LE can be ascribed to a corresponding increase in muscular demand elicited by an increased rate of muscle contraction. It has been suggested that, as the speed of contraction increases, an increase in internal muscular work is required to overcome the augmented intramuscular friction. ${ }^{2}$ It has also been speculated that, as pedal frequency increases, the recruitment of additional motor units increases despite an unchanging workload. ${ }^{40}$ This may be supported by the study of MacIntosh et al, ${ }^{19}$ who found increased electromyographic activity in the gastrocnemius muscle as pedal frequency increased from 40 to $80 \mathrm{rev} / \mathrm{min}$ during cycling at a constant power output. Power output is the product of pedal frequency and brake resistance. From the present findings, it appears that the velocity at which muscle contracts is a more important determinant of energy demand of a given activity, and this relation between velocity and energy expenditure remains similar across different exercise modalities or relative exercise intensities.

Whereas greater energy expenditure was seen at $80 \mathrm{rev} /$ min during both UE and LE, the energy distribution between carbohydrate and fat oxidation differed between the two modes of exercise. During UE, as the total energy expenditure was from carbohydrate and fat oxidation, both these subcomponents remained the same across the three pedal rates. However, during LE, the increase in energy expenditure was accompanied by a corresponding increase in carbohydrate oxidation, whereas fat oxidation remained essentially unchanged. This increase in the amount of energy derived from carbohydrate during LE may simply be the consequence of an increase in $\mathrm{VO}_{2}$ or exercise intensity as a result of an increase in pedal frequency. This finding is consistent with the observation by Hoelting et $a^{20}$ of a reduction in leg blood flow during knee extension at 80 contractions per minute compared with 40 contractions per minute. This reduced circulation in the exercising limbs may force the muscle to depend more on its own glycogen to yield energy. The authors of the previous studies, which used much higher power outputs, argued that most of the additional motor units recruited at higher pedal frequencies probably consist primarily of fast twitch muscle fibres, which are more carbohydrate dependent. ${ }^{4} 10$ This, however, does not explain why more energy was derived from carbohydrate, as the
Take home message

Despite unchanging power output, exercise performed at a greater speed elicits greater energy expenditure. This is of pertinence to those who use exercise apparatus that allows selection of speed of movement without a concurrent change in workload.

intensity used during LE was only about $40 \% \mathrm{Vo}_{2}$ peak and did not reach the threshold for mobilising much of the fast twitch muscle fibre of the exercising muscle. ${ }^{21}$

Failure to observe a similar increase in carbohydrate oxidation at $80 \mathrm{rev} / \mathrm{min}$ during UE may be due to the higher RERs observed at 40 and $60 \mathrm{rev} / \mathrm{min}$. In other words, when the rate of carbohydrate utilisation was calculated, the lower level of energy expenditure achieved at these lower pedal rates may have been offset by the higher percentage of energy derived from carbohydrate, as determined from the RER. The lower RERs found at $80 \mathrm{rev} / \mathrm{min}$ were somewhat unexpected as it was our hypothesis that this speed would be associated with an increase in recruitment of fast twitch muscle fibres and thus utilisation of carbohydrate. The reason why RER responded differently during UE is not readily apparent. The relative intensity achieved during UE corresponded to nearly $70 \%$ of mode specific $\mathrm{VO}_{2}$ peak, and this percentage was much higher than that during LE. As higher RERs at lower pedal rates were also observed during cycle exercise by Hagan et al, ${ }^{10}$ who used a much higher workload, it is tempting to speculate that such reversed RER responses during UE may be because the relative intensity experienced was much greater during UE than LE. A lower pedal rate is associated with a greater brake resistance, which dictates the level of muscular tension developed. It is possible that, when exercise is performed at a high intensity, an increase in muscular tension stimulates carbohydrate utilisation to a similar or even greater extent than an increase in pedal frequency. This contention is underscored by Ahlquist et al, ${ }^{11}$ who showed greater glycogen utilisation of type II muscle fibres after cycle exercise of $85 \%$ $\mathrm{VO}_{2}$ max at 50 than at $100 \mathrm{rev} / \mathrm{min}$.

In conclusion, we found that pedalling at a greater frequency helped to maximise energy expenditure during either UE or LE despite an unchanging power output and a concomitant reduction in brake resistance. This increase in energy expenditure was brought about by a corresponding increase in carbohydrate oxidation during LE when the intensity was rather low. The fact that no differences in either carbohydrate or fat oxidation were observed for the three pedal rates during UE when the intensity was higher suggests that these speed induced changes in carbohydrate utilisation seen during LE can be altered by exercise modality or relative exercise intensity.

\section{Authors' affiliations}

J Kang, J R Hoffman, M Wendell, H Walker, M Hebert, The College of New Jersey, Ewing, NJ, USA

\section{REFERENCES}

1 Croisant PT. Effect of pedal rate, brake load and power on metabolic responses to bicycle ergometer work. Ergonomics 1984;27:691-700.

2 Donovan CM, Brooks GA. Muscular efficiency during steady-state exercise: effects of speed and work rate. J Appl Physiol 1977;43:431-9.

3 Faria I, Sjojaard G, Bonde-Petersen F. Oxygen cost during different pedaling speeds for constant power output. J Sports Med 1982;22:295-9.

4 Gaesser GA, Brooks GA. Muscular efficiency during steady-rate exercise: effect of speed and work rate. J Appl Physiol 1975;38:1132-9.

5 Merrill EG, While JA. Physiological efficiency of constant power output at varying pedal rates. J Sports Sci 1984;2:25-34. 
6 Power SK, Beadle RE, Mangum M. Exercise efficiency during arm ergometry effects of speed and work rate. J Appl Physiol 1984;56:495-9.

7 Robertson R, Goss F, Michael T, et al. Metabolic and perceptual responses during arm and leg ergometry in water and air. Med Sci Sports Exerc 1995:27:760-4

8 Woolford SM, Withers RT, Craig NP, et al. Effect of pedal cadence on the accumulated oxygen deficit, maximal aerobic power and blood lactate transition thresholds of high-performance junior endurance cyclists. Eur J Appl Physiol 1999;80:285-91.

9 Buchanan $\mathrm{M}$, Weltman A. Effect of pedal frequency on $\mathrm{VO}_{2}$ and work output at lactate threshold (LT), fixed blood lactate concentrations, of $2 \mathrm{mM}$ and $4 \mathrm{mM}$, and max in competitive cyclists. Int J Sports Med 1985;6:163-8.

10 Hagan RD, Weis SE, Raven PB. Effect of pedal rate on cardiorespiratory responses during continuous exercise. Med Sci Sports Exerc 1992;24:1088-95.

11 Ahlquist LE, Bassett Jr DR, Stufit R, et al. The effect of pedal frequency on glycogen depletion rates in type I and type II quadriceps muscle fibers during submaximal cycling exercise. Eur J Appl Physiol 1992;65:360-4.

12 Gollnick PD, Armstrong RB, Saubert IV CW, et al. Enzyme activity and fiber composition skeletal muscle of untrained and trained men. J Appl Physiol 1972;33:312-19.
13 Johnson MA, Polgar J Weightman D, etal. Data on the distribution of fiber types in thirty-six human muscles: an autopsy study. J Neurol Sci 1973;18:111-29.

14 Kang J, Chaloupka EC, Mastrangelo MA, et al. Physiological responses to upper body exercise on an arm and a modified leg ergometer. Med Sci Sports Exerc 1998:31:1453-9.

15 Kang J, Robertson RJ, Goss FL, et al. Metabolic efficiency of arm cranking and leg cycling at the same mode-specific relative exercise intensity. Med Sci Sports Exerc 1997;29:377-82.

16 McArdle WD, Katch FI, Katch VL. Measurement of human energy expenditure. In: Exercise physiology textbook, 2nd ed. Philadelphia: Lea \& Febiger, 1986:121-30.

17 Sawka MN, Foley ME, Pimental NA, et al. Determination of maximal aerobic power during able-body exercise. J Appl Physiol 1983;54:113-17.

18 Åstrand PO. Work test with the bicycle ergometer. Varberg, Sweden: AB Cykelfabriken Monark, 1965

19 Macintosh BR, Neptune RR, Horton JF. Cadence, power, and muscle activation in cycle ergometry. Med Sci Sports Exerc 2000;32:1281-7.

20 Hoelting BD, Scheuermann BW, Barstow TJ. Effect of contraction frequency on leg flow during knee extension exercise in humans. J Appl Physiol 2001;91:671-9.

21 Sale DG. Influence of exercise and training on motor unit activation. Exerc Sport Sci Rev 1987; 15:95-151.

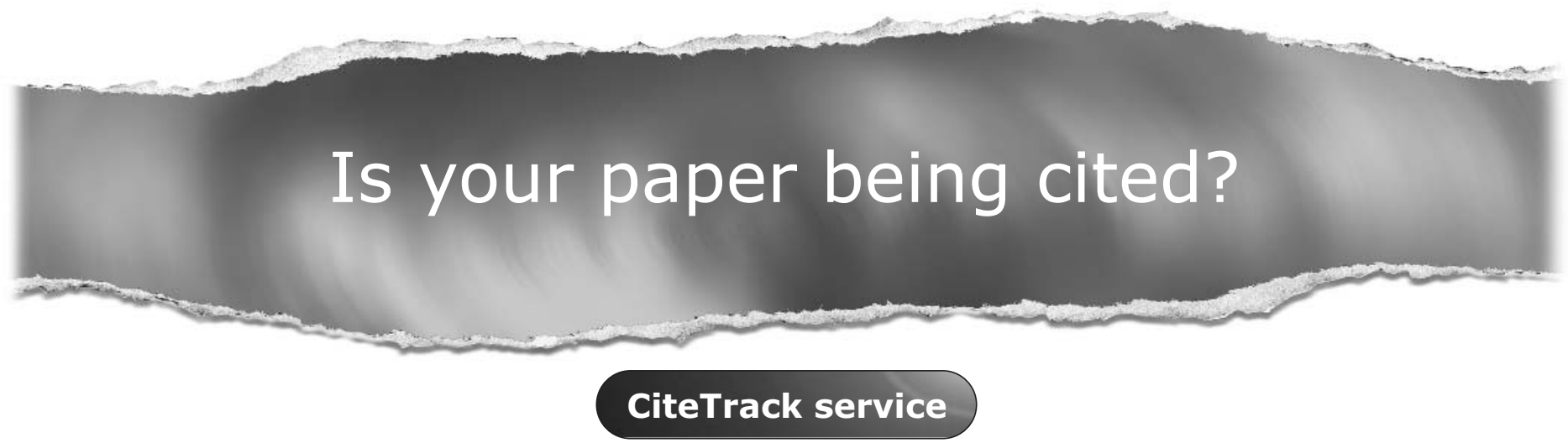

CiteTrack will alert you by email whenever new content in the British Journal of Sports Medicine or a participating journal is published that matches criteria you want to track

Topics: Tell CiteTrack which words or subjects to watch for in new content Authors: Be alerted whenever key authors you are following publish a new paper Articles: Know whenever a paper of interest to you is referenced by another paper

www.bjsportmed.com 